\title{
Plaque Hypothesis - Change that Ever Change?
}

\author{
Maria Subash Aaron Muthuraj* \\ Sri Ramakrishna Dental College and Hospital, Coimbatore, Tamil Nadu, India \\ *Corresponding Author: Maria Subash Aaron Muthuraj, Sri Ramakrishna Dental College and Hospital, Coimbatore, Tamil Nadu, India.
}

Received: September 24, 2019; Published: November 01, 2019

DOI: $10.31080 / A S D S .2019 .03 .0688$

Dental plaque is a major etiological agent for gingivitis and periodontitis. A hypothesis on the dental plaque was first given by Miller [1]. In his "Nonspecific plaque hypothesis", he hypothesized that the severity of the disease depends upon the amount of plaque accumulation. Even though it is an old concept, most of our periodontal treatment strategies still depend on it. By the 1960s with improvement in microscopic imaging techniques, microbial patterns were found to be highly varying between healthy and diseased individuals, giving rise to "Specific plaque hypothesis" [2]. During this period major developments in growth and isolation of bacteria such as Aggregatibacter actinomycetemcomitans had occurred. At that period, the treatment modality for periodontitis shifted towards using antibiotics as an adjuvant to therapy to target specific bacteria. But the major drawback of the above hypotheses was that they hadn't supported the multifactorial nature of periodontitis. To overcome the drawbacks of the above hypotheses Marsh PD gave the "Ecological Plaque Hypothesis"[3]. He hypothesized that plaque independent factors such as immune disorders, hormonal imbalance, stress, smoking, etc. will create an environment conducive to pathogenesis and disease progression. During this period host modulating agents as well as antioxidants had been added to the periodontal treatment modalities. Hajishengallis gave "Keystone pathogenesis" which hypothesized that Porphyromonas gingivalis is a key pathogen that can initiate the disease on its presence and facilitate the progression of the disease through its array of virulence factors [4]. But the involvement of potential organisms such as Filifactor alocis and Epstein barr virus hadn't taken into account. Viruses had been identified as an etiologic agent in periodontitis; its re-activation theory supports the burst activity of periodontitis which validates the use of antiviral drugs as an adjuvant [5].

As periodontal microbiota includes bacteria, virus, fungi, archaea and protozoa also, every possible organism should be considered rather than on focusing on a particular organism. Hajishengallis again gave a concept of "Polymicrobial synergy and dysbiosis" in the next year which hypothesized that different members and specific gene combinations converge to shape and stabilize a disease provoking microbiota [6]. But the red complex organisms and environmental factors are not given much consideration. Every plaque hypothesis had an acceptable and unacceptable ideology. Comprehensive plaque hypothesis combining positive points from the previous hypothesis and incorporating newer valid research is required now, to eradicate the demerits of previous plaque hypotheses and finalize the factors influencing change in harmless microbiota to harmful microbiota during the progression of the disease. As periodontitis is one of the widely prevalent oral diseases involving the world population we have to put an end to change in hypothesis and derive a definite etiology and treatment for it.

\section{Bibliography}

1. Miller WD. "The Micro-Organisms of the Human Mouth". Philadelphia, PA: The S.S. White Dental MFG. Company (1890).

2. Loe H., et al. "Experimental gingivitis in man". Journal of Periodontology 36 (1965): 177-187.

3. Marsh PD. "Microbial ecology of dental plaque and its significance in health and disease". Advances in Dental Research 8 (1994): 263-271.

4. Hajishengallis G., et al. "Low-abundance biofilm species orchestrates inflammatory periodontal disease through the commensal microbiota and complement". Cell Host and Microbe 10 (2011): 497-506.

5. Slots J. "Human viruses in periodontitis". Periodontology 53 (2010): 89-110.

6. Hajishengallis G and Lamont RJ. "Beyond the red complex and into more complexity: The polymicrobial synergy and dysbiosis (PSD) model of periodontal disease etiology". Molecular Oral Microbiology 27 (2012): 409-419.

\section{Volume 3 Issue 12 December 2019 \\ (C) All rights are reserved by Maria Subash Aaron Muthuraj.}

